\title{
Tests from Proofs
}

\author{
Patrice Godefroid \\ Microsoft Research \\ pg@microsoft.com
}

\begin{abstract}
Test generation has recently become the largest application of SMT solvers as measured by computational usage. At Microsoft, the Z3 SMT solver has solved more than 200 million constraints over the last two years as a component of the whitebox fuzzer SAGE. Whitebox fuzzing extends dynamic test generation based on symbolic execution and constraint solving from unit testing to whole-application security testing. Since 2009, SAGE has been running non-stop on (average) 100+ machines automatically "fuzzing" hundreds of applications in a dedicated lab owned by the Microsoft Windows security test team. In the process, SAGE found many new security vulnerabilities (missed by blackbox fuzzing and static program analysis) and was credited to have found roughly one third of all the bugs discovered by file fuzzing during the development of Microsoft's Windows 7 , saving millions of dollars by avoiding expensive security patches to nearly a billion PCs.

In the second part of the talk, I will present a new form of test generation, named higher-order test generation, where imprecision in symbolic execution is represented using uninterpreted functions in logic path constraints. I will explain why such functions need be universally quantified, hence requiring tests to be generated from validity proofs of first-order logic formulas, rather than from satisfiability proofs of quantifier-free first-order logic formulas as usual.
\end{abstract}

